\title{
A Case of Localized Psoriasis Following Treatment with lodine131 for Hyperthyroidism: A Rare Entity
}

\author{
(1) Aniruddha Mandal, (1) Pramit Nandy, (1) Subhasmita Baisya, (1) Subhadeep Mallick, (1) Olympia Rudra
}

Institute of Post Graduate Medical Training \& Research and SSKM Hospital, Clinic of Dermatology, Kolkata, India

\section{ABSTRACT}

Psoriasis is a T-cell mediated inflammatory skin disease, characterized by circumscribed erythematous plaques, covered by silvery micaceous scales. It is known to be triggered by a number of factors including drugs. Drugs may either cause de novo psoriasis or responsible for aggravating preexisting psoriasis. The morphological types can vary from localized or generalized plaque psoriasis to pustular psoriasis and even erythroderma. In this work, we report the case of a 45-year-old male patient who developed localized psoriasis following treatment with iodine $^{131}$ for hyperthyroidism. Based on history, clinical pictures, localized examination and histopathological findings with psoriasiform changes favoured the diagnosis. He was treated with topical steroid with clobetasol propionate (0.05\%) and salicylic acid (6\%) combination for 1 month following which lesions resolved. We suspected that the mechanism behind this is due to activation of dihydrofolate reductase by radioactive iodine. Further study about the folic acid pathway in psoriasis and the connection between radioactive iodine with psoriasis may be necessary.

Keywords: Localized psoriasis, Radioactive iodine ${ }^{131}$, Hyperthyroidism

\section{Introduction}

Psoriasis is a T-cell mediated inflammatory skin disease, characterized by circumscribed, erythematous well-defined plaques covered by silvery micaceous scales. Radioactive iodine (1131) is an important isotope used in hyperthyroidism (Graves' disease, Toxic multinodular goiter and autonomously functioning thyroid nodule). The most significant cutaneous adverse effect of 1131 is iododerma. Here we report a case of localized psoriasis following treatment with radioactive iodine for hyperthyroidism.

\section{Case Report}

A 45 years old male shopkeeper by occupation attended skin OPD with itchy plaque over dorsum of feet which was gradually increasing for last 1 month. He is a known case of thyrotoxicosis for which he was on oral carbimazole for last 6 years and not responding and recently treated with 9.2 mili curie of oral radioiodine ${ }^{131}$. He developed erythematous papular eruption over dorsum of both feet after 20 days of 1131 therapy and it is slowly increasing in size. On examination symmetrical hyperkeratotic plaque was present over dorsum of both feet (Figure 1). Auspitz's sign was positive. Oral radioiodine therapy had been stopped. No others cutaneous or mucosal sites were involved. He had no history of similar episode in the past. General, systemic and routine blood examinations were unremarkable other than thyroid profile. Histopathology showed hyperkeratosis, parakeratosis acanthosis, suprapapillary thinning, club shaped rete ridges and collection of neutrophils in stratum corneum (Munro's microabscess) and in dermis there was dilated blood vessels and perivascular lymphocytic infiltrates in papillary dermis (Figures 2, 3). A final diagnosis of 1131 induced localized plaque psoriasis was made. Patients was treated with topical steroid with clobetasol propionate (0.05\%) and salicylic acid (6\%) 


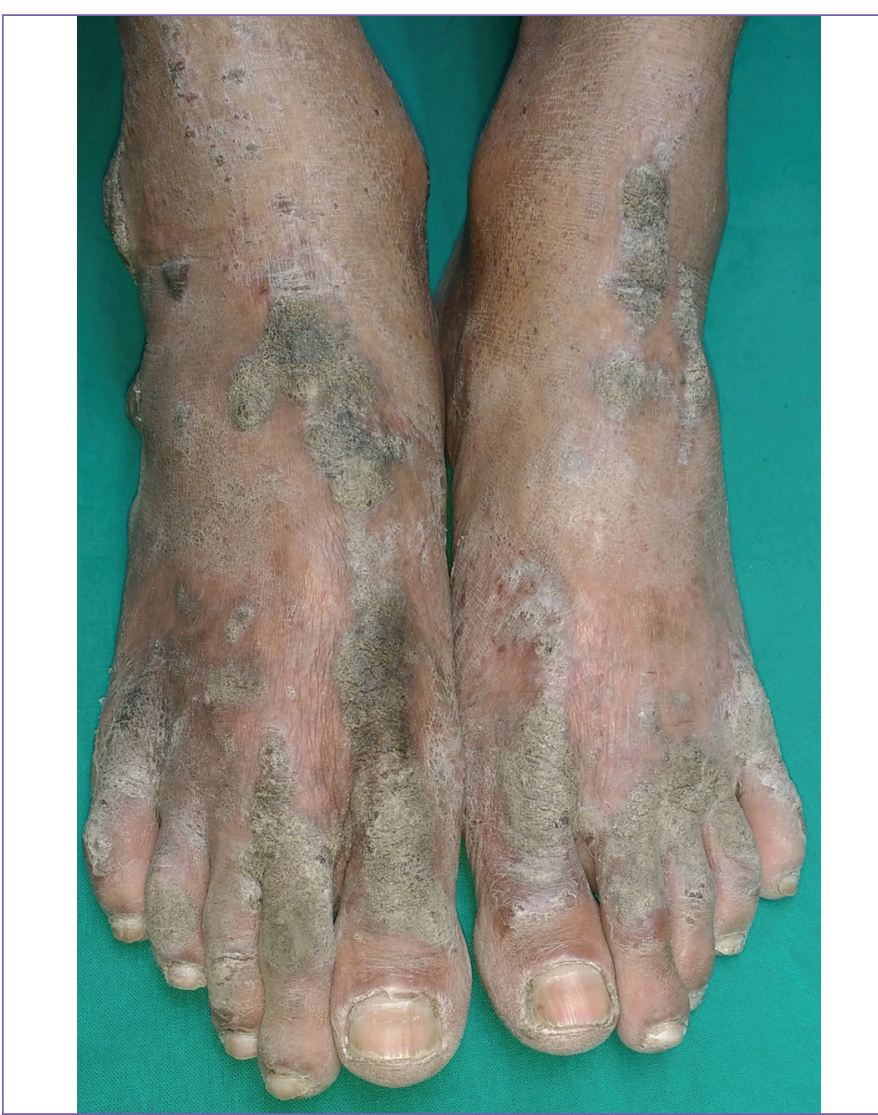

Figure 1. Symmetrical hyperkeratotic plaque was present over dorsum of both feet

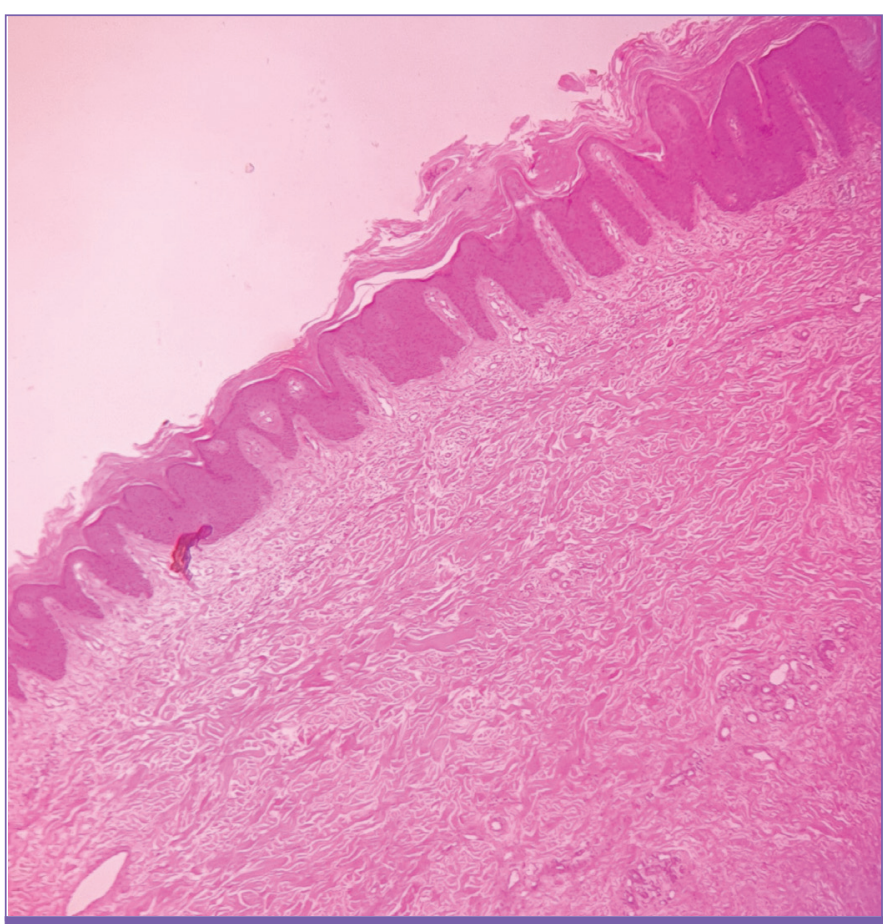

Figure 2. Hyperkeratosis, parakeratosis acanthosis, suprapapillary thinning, club shaped rete ridges (hematoxylin and eosin stain under $4 \mathrm{x}$ ) combination for 1 month following which lesions resolved and there was only hyper pigmentation (Figure 4). Radioiodine therapy had not been restarted and he had been started on oral methimazole. Informed consent has been taken from the patient.

\section{Discussion}

Psoriasis is a T-cell mediated common, chronic and recurrent inflammatory skin disease, characterized by circumscribed, erythematous sharply demarcated papules and plaques covered by silvery micaceous scales. Many factors contribute its pathogenesis like genetic, immunological and environmental factors. Several other factors like trauma, stress, infections and medications might exacerbate psoriasis [1]. Radioactive $\mathrm{I}^{131}$ is an important isotope used in hyperthyroidism (Graves' disease, Toxic multinodular goiter and autonomously functioning thyroid nodule). Cutaneous adverse effect of 131 include iododerma, which is characterized by acneiform eruption with inflammatory follicular pustules, or may present as urticaria or bullous lesion with ulceration and crust. lododerma may occurs in the face, neck, extremities and trunk [2]. There have been many drugs documented to directly trigger the eruption of psoriasis (antibiotics, non-steroidal anti-inflammatory drugs, angiotensin-converting enzyme inhibitors, interferon, amiodarone,

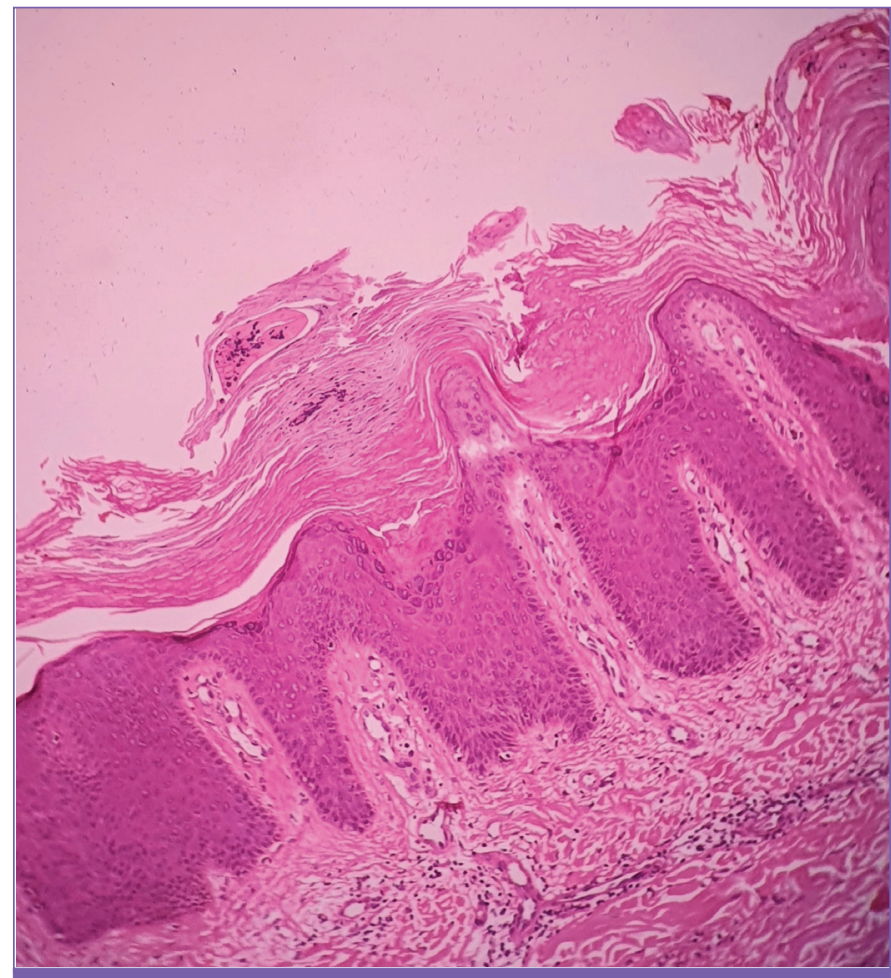

Figure 3. Hyperkeratosis, parakeratosis acanthosis, suprapapillary thinning, club shaped rete ridges and collection of neutrophils in stratum corneum (Munro's microabscess) and in dermis there was dilated blood vessels and perivascular lymphocytic infiltrates in papillary dermis (hematoxylin and eosin stain under 10x) 


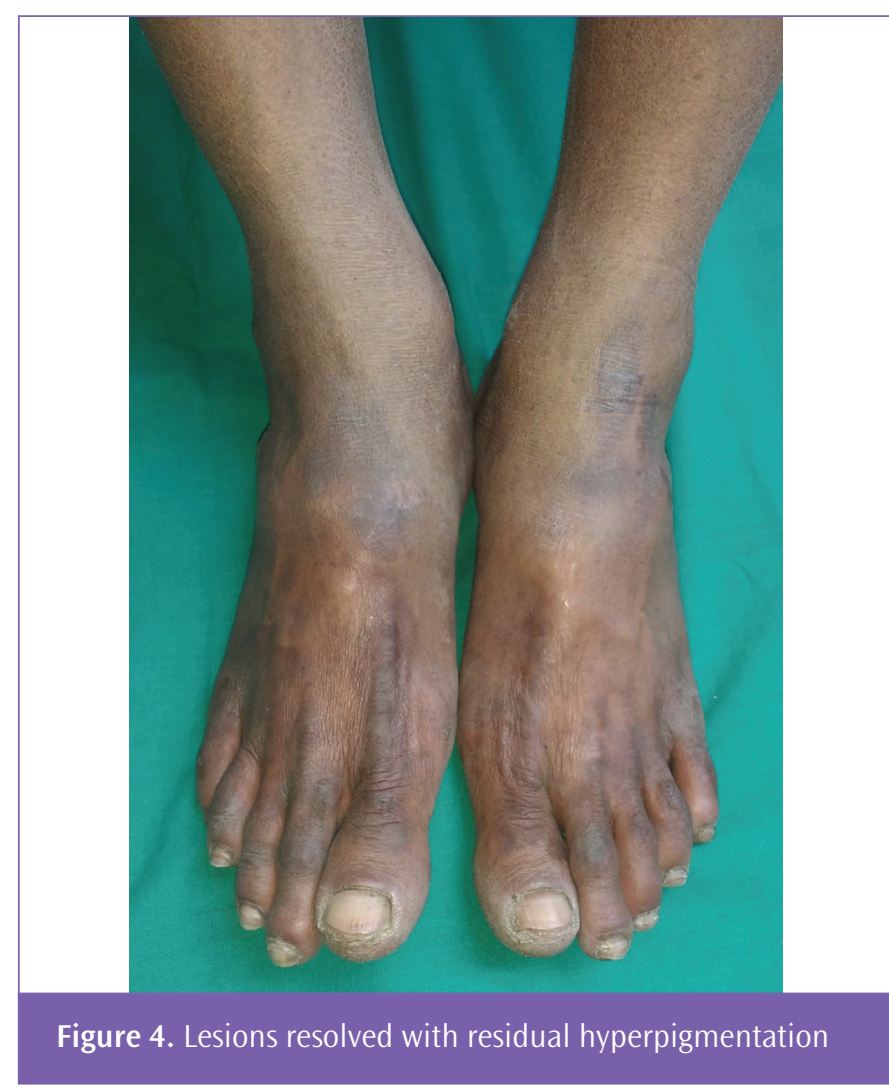

terbinafine, benzodiazepines, digoxin, clonidine, quinidine, gold, potassium iodide, imiquimod etc.) and many others exacerbate the existing psoriasis (acetazolamide, aminoglutethimide, amiodarone, antibiotics, terbinafine, diltiazem, hydroxychloroquine, lithium, potassium iodide, propanol etc.) [3,4]. It is very difficult to explain the exact mechanism, they may affect the psoriatic process at different stages but with same results. It has been seen that iodine/iodides specifically activate the enzyme dihydrofolate reductase and Kang and Kim [5] reported a case of psoriasis exacerbated by radioactive iodine therapy. We suspected that the mechanism behind this, is due to activation of dihydrofolate reductase through radioactive iodine as there is no other significant drug history. Further study about the folic acid pathway in psoriasis and the connection between radioactive iodine with psoriasis may be necessary. This article has been presented to highlight the rarity of such condition.

\section{Ethics}

Informed Consent: Informed consent has been taken from the patient.

Peer-review: Internally peer-reviewed.

\section{Authorship Contributions}

Surgical and Medical Practices: A.M., P.N., S.B., S.M., O.R., Concept: A.M., P.N., S.B., S.M., O.R., Design: A.M., P.N., S.B., S.M., O.R., Data Collection or Processing: A.M., P.N., S.B., S.M., O.R., Analysis or Interpretation: A.M., P.N., S.B., S.M., O.R., Literature Search: A.M., P.N., S.B., S.M., O.R., Writing: S.B.

Conflict of Interest: No conflict of interest was declared by the authors.

Financial Disclosure: The authors declared that this study received no financial support.

\section{References}

1. Burden AD, Kirby B. Psoriasis and related disorders. In: Griffiths CEM, editor Rook's Textbook of Dermatology. 9th ed. West Sussex: John Wiley \& Sons 2016. p. 1130-1180.

2. Vandergriff TW, Yancey KB. Iododerma following radioactive iodine ablation of the thyroid or Grave's disease. J Drugs Dermatol 2011;10:1070-1071.

3. Milavec-Puretić V, Mance M, Ceović R, Lipozenčić J. Drug induced psoriasis. Acta Dermatovenerol Croat 2011;19:39-42

4. Litt JZ. Drug Eruption Reference Manual, 12th ed. London, New York: Taylor and Francis; 2006. p. 643-645.

5. Kang YS, Kim TY. A case of psoriasis exacerbated by radioactive iodine therapy. Ann Dermatol 2014;26:785-786. 